Background Several studies have shown an association between low 25-hydroxy-vitamin D levels $(25(\mathrm{OH}) \mathrm{D})$ and increased prevalence of metabolic syndrome (MetS), total adiposity and insulin resistance in obese and patients with type 2 diabetes.

Objective The aim of this study was to investigate the association between $25(\mathrm{OH}) \mathrm{D}$ levels and insulin resistance markers in adolescents with policystic ovary syndrome (PCOS) and to show changes in these parameters after 3 months treatment with 4000 $\mathrm{IU} / \mathrm{d}$ vit D3.

Methods We determined body mass index (BMI), body fat percentage $(\mathrm{BF} \%)$ and serum $25(\mathrm{OH}) \mathrm{D}$, leptin/adiponectin ratio, HOMA-IR index, HDL, triglycerides, FSH, LH and free testosteron (fT) levels in 51 girls with PCOS (mean age 16,8 $\pm 1,1$ year) at the admission and 3 months after vit $\mathrm{D}$ therapy.

Results All of the girls had insufficient levels of 25(OH)D (less than $25 \mathrm{mg} / \mathrm{ml}) .25(\mathrm{OH}) \mathrm{D}$ levels were negatively correlated with BMI $(r=-0,262)$, BF\% ( $r=-0,232)$, HOMA-IR $(r=-0,195)$, leptin $(r=-0,283)$ and triglycerides $(r=-0,189)$, and positively correlated with adiponectin $(\mathrm{r}=0,264)$, and HDL levels $(\mathrm{r}=$ $0,258)$, all $\mathrm{p}<0,05$.

3 months after therapy 25(OH)D levels increased from 17.3 $\pm 6.3 \mathrm{ng} / \mathrm{ml}$ to $39.1 \pm 9.2 \mathrm{ng} / \mathrm{ml}(\mathrm{p}=0.029)$, HOMA-IR decreased from $4.98 \pm 0.42$ to $3.35 \pm 0.45$, leptin/adiponectin ratio from $7.39 \pm 0.03$ to $5.98 \pm 0.03$ and triglycerides levels from $2.32 \pm 0.32$ to $1.68 \pm 0.27(\mathrm{p}=0.009)$. There were no significant changes in BMI, BF\%, HDL, FSH, LH and fT levels. Conclusions There is a significant association between low 25 $(\mathrm{OH}) \mathrm{D}$ levels and insulin resistance markers in adolescents with PCOS. Correcting 25(OH)D levels improves insulin sensitivity but does not improve hyperandrogenism and LF/FSH ratio in adolescents with PCOS.

\section{PS-008 PHYSICAL ACTIVITY AND SCREEN TIME GUIDELINES FOR PRESCHOOLERS: THE RELATIONSHIP TO INFORMATION SOURCES}

${ }^{1} \mathrm{~A}$ Pujadas Botey, ${ }^{2} \mathrm{C}$ Bukutu, ${ }^{3}$ WB Gibbard, ${ }^{3} \mathrm{~K}$ MacLellan, ${ }^{4} \mathrm{H}$ Bayrampour, ${ }^{4} \mathrm{~A}$ Vinturache ${ }^{5}$ D Slater, ${ }^{6}$ R Breitkreuz, ${ }^{6}$ L Sakaluk-Moody, ${ }^{7}$ S Lynch, ${ }^{8}$ S Tough. ${ }^{1}$ Alberta Centre for Child Family and Community Research, Calgary, Canada; ${ }^{2}$ Child and Younth Data Laboratory, Alberta Centre for Child Family and Community Research, Edmonton, Canada; ${ }^{3}$ Department of Paediatrics, University of Calgary and Alberta Health Services, Calgary, Canada; ${ }^{4}$ Department of Paediatrics, University of Calgary, Calgary, Canada; ${ }^{5}$ Department of Pharmacology and Therapeutics, University of Calgary, Calgary, Canada; ${ }^{6}$ Department of Human Ecology, University of Alberta, Edmonton, Canada; ${ }^{7}$ Community University Partnership, University of Alberta, Edmonton, Canada; ${ }^{8}$ Department of Paediatrics Department of Community Health Sciences, Alberta Centre for Child Family and Community Research and University of Calgary, Calgary, Canada

\subsection{6/archdischild-2014-307384.303}

Background Evidence to date suggests that a trajectory for better child outcomes may begin with parents' knowledge of healthy habits for their children. Guidelines about preschool engagement in physical activity and screen time are posted on government websites and are accessible through communitybased settings to inform parents. The relationship between use of these resources and uptake of information is unknown.

Study Questions/aims (1) What resources do parents use to access information about their children's health? (2) What are the characteristics of parents using government-regulated resources versus other resources? (3) Does type of resource used influence family practices related to physical activity and screen time with preschool children?
Methods 736 randomly selected parents in Alberta, Canada, completed a telephone survey exploring: child-health-related topics of interest, resources accessed, screen time and active living habits. Data was analysed using univariate and multivariate techniques $(\mathrm{p}<0.05)$.

Results $62 \%$ of parents were interested in accessing information about their children's physical health. $80 \%$ used governmentregulated resources. Over half of children aged 2 to 4 did not reach the 3-hours daily minimum of physical activity (55-66\%) and similar percentages exceeded the 1-hour daily maximum screen time recommended (50-76\%). Parents using governmentregulated resources were not more likely than other parents to follow physical activity and screen time recommendations.

Conclusions Strategies to improve uptake of physical activity and screen time guidelines may require more than information exchange through the internet or in community-based settings. Optimal child health and development may be enhanced through better uptake of guidelines.

\section{PS-009 AMBULATORY BLOOD PRESSURE MEASUREMENT IN ADOLESCENTS WITH CARDIOVASCULAR RISK: TYPE 1 DIABETIC PATIENTS VERSUS OVERWEIGHT SUBJECTS}

${ }^{1} \mathrm{~V}$ Giacchi, ${ }^{1} \mathrm{~T}$ Timpanaro, ${ }^{2} \mathrm{D}$ Lo Presti, 'S Passanisi, ${ }^{1} \mathrm{C}$ Mattia, ${ }^{1} \mathrm{P}$ Betta, ${ }^{1} \mathrm{C}$ Grasso, ${ }^{2} \mathrm{M}$ Caruso, ${ }^{3} \mathrm{P}$ Sciacca. ${ }^{1}$ Pediatric Department, AOU Policlinico-Vittorio Emanuele, Catania, Italy; ${ }^{2}$ Pediatric Endocrinology Clinic, AOU Policlinico-Vittorio Emanuele, Catania, Italy; ${ }^{3}$ Pediatric Cardiology Clinic, AOU Policlinico-Vittorio Emanuele, Catania, Italy

\subsection{6/archdischild-2014-307384.304}

\section{Abstract}

Background and aim Adolescents with type 1 diabetes (T1DM) and obesity present higher cardiovascular risk and ambulatory blood pressure measurements (ABPM) can identify the non-dipper status, often the first sign of hypertension. The aim of our observational cross-sectional case-control study conducted in T1DM, overweight and healthy adolescents was to assess blood pressure (BP) parameters to identify subclinical cardiovascular risk.

Methods We performed ABPM for $24 \mathrm{~h}$ in 39 adolescents (26 male and 13 female) with T1DM followed in our Paediatric Department between January 2011 and December 2012. We compared the data of patients with those of overweight subjects and healthy controls.

Results ABPM revealed no significant difference between T1DM patients and overweight subjects except for night-time diastolic $\mathrm{BP}$ values $(\mathrm{p}=0.000)$. Instead we found significant difference between type 1 diabetic patients and healthy controls in all $24 \mathrm{~h}$ Systolic $(\mathrm{p}=0.000), 24 \mathrm{~h}$ Diastolic $(\mathrm{p}=0.002)$, Day-time Systolic $(p=0.005)$, Night-time Systolic $(p=0.000)$, Day-time diastolic $(\mathrm{p}=0.038)$ and Night-time Diastolic (0.000) BP values. We detected hypertension in 10/39 (25.6\%) T1DM patients and in $8 / 39(20.5 \%)$ overweight subjects ( $p=0.591)$, whereas noone of healthy controls presented hypertension $(\mathrm{p}=0.001)$. We observed non-dipper pattern in 32/39 (82\%) T1DM patients, in $26 / 39(66.6 \%)$ overweight subjects $(\mathrm{p}=0.120)$, and in $13 / 39$ $(33.3 \%)$ of healthy controls $(p=0.000)$.

Conclusions ABPM studies allows to identify patients at risk for the development of hypertension, who might benefit from the early introduction of anti-hypertensive therapies. In evaluating $\mathrm{BP}$ in T1DM and overweight subjects, ABPM should be used since a reduced dipping can indicate incipient hypertension. 